\title{
Sleep fragmentation and working memory in healthy adults
}

Masato Okuda ${ }^{1}$ Akiko Noda ${ }^{1 *}$

Sho Mabuchi ${ }^{2}$

Kunihiro Iwamoto ${ }^{3}$

Masahiro Banno ${ }^{3,4}$

Seiko Miyata ${ }^{3}$

Fumihiko Yasuma ${ }^{5}$

Norio Ozaki ${ }^{3}$

${ }^{1}$ Chubu University Graduate School of Life and Health Sciences, Department of Biomedical Sciences - Kasugai - Aichi Japan.

${ }^{2}$ Chubu University Collage of Life and Health Sciences, Department of Biomedical Sciences -Kasugai - Aichi- Japan.

${ }^{3}$ Nagoya University Graduate School of Medicine, Department of Psychiatry -

Nagoya - Aichi -Japan.

${ }^{4}$ Seichiryo Hospital, Department of

Psychiatry - Nagoya - Aichi - Japan.

${ }^{5}$ National Hospital Organization Suzuka

Hospital, Department of Internal

Medicine - Suzuka - Mie -Japan.

\footnotetext{
*Corresponding author:

Akiko Noda

E-mail: anoda@isc.chubu.ac.jp
}

Received: September 3, 2020; Accepted: December 8, 2020.

DOI: $10.5935 / 1984-0063.20200088$

\begin{abstract}
Introduction: Sleep is essential for performing cognitive function in humans. We have hypothesized that sleep fragmentation compared to sleep efficiency may have a negative impact on the working memory. Material and Methods: Twenty-eight healthy adults (18 males and 10 females; mean age $27.8 \pm 15.5$ years) were enrolled in this study. We measured the total sleep time (TST), sleep efficiency, \%stage wakefulness (W), \%stage rapid eye movement (REM), \%stage N1, \%stage N2, $\%$ stage N3, wake after sleep onset (WASO), and arousal index using polysomnography. Working memory, executive function, and sustained attention of three domains of cognitive function were evaluated with the number of back task (N-back task), Wisconsin card sorting test (WCST), and continuous performance test-identical pairs (CPT-IP), respectively. Results: The percentage of correct answers on the 2-back task was significantly correlated with \%stage REM, \%stage N1, and \%stage N2 (\%stage REM: $r=0.505, p=0.006$; \%stage N1: $r=-0.637, p<0.001 ; \%$ stage N2: $r=0.670$, $p<0.001)$, and multiple regression analysis including the stepwise forward selection method revealed that \%stage N2 was the most significant factor (\%stage N2: $\beta=0.670, p<0.001)$. The percentage of correct answers on the 2-back task was also significantly correlated with TST, sleep efficiency, WASO, and arousal index (TST: $r=0.492, p=0.008$; sleep efficiency: $r=0.622, p<0.001$; WASO: $r=-$ $0.721, p<0.001$; arousal index: $r=-0.656, p<0.001)$, and WASO was the significant factor $(\beta=-2.086$, $p=0.007)$. The WCST category achievement and CPT-IP $d$-prime score were correlated with none of the sleep variables. Conclusion: Increased WASO and a decrease in \%stage N2 were associated with worse working memory.
\end{abstract}

Keywords: Sleep Fragmentation; Sleep Stage; Wake After Sleep Onset; Working Memory; Cognitive Function. 


\section{INTRODUCTION}

Sleep plays an important role in mediating multiple domains of cognitive function in humans ${ }^{1}$. Sleep restriction is a facet of modern life that jeopardizes the cognitive performance including lapses of attention, slowed working memory, reduced cognitive throughput, and perseveration of thought ${ }^{2}$. An epidemiological study demonstrated that short and long sleep duration were associated with both objectively assessed and self-reported decreases in cognitive function in the general population ${ }^{3}$. In particular, the adverse effects of a short sleep duration on cognitive function have been well studied ${ }^{2-5}$. However, there has been no consensus regarding the impacts of sleep structure and sleep fragmentation on working memory.

The two main sleep states, i.e., the non-rapid eye movement (NREM) stages of N2 and N3 and rapid eye movement (REM) stage, have specific roles in sleep-dependent memory processing. Stage N3 and stage REM, as well as stage N2, are involved in memory consolidation ${ }^{6}$. Regarding the specific patterns of neural oscillation associated with each sleep stage, the pontogeniculo-occipital waves and theta rhythms in stage REM, sleep spindles in stage N2, and slow wave activity of stage N3 have been postulated ${ }^{7,8}$. Moreover, an improvement in the visual discrimination task is correlated with the levels of stage REM ${ }^{9}$, while an improvement in the finger-tapping task is correlated with stage $\mathrm{N} 2{ }^{10}$. Although sleep fragmentation owing to frequent arousals during sleep likely contributes to the increased risk of cognitive decline, the impact of frequent arousals from sleep on cognitive function has not been systematically analyzed.

We previously showed that a short sleep duration had a negative impact on three domains of cognitive function, namely working memory, executive function, and sustained attention in young adults ${ }^{11-13}$ using the number of back task (N-back task) ${ }^{14,15}$, Wisconsin card sorting test (WCST) ${ }^{16}$, and continuous performance test-identical pairs (CPT-IP) ${ }^{17}$, respectively. The behavioral, cognitive, and psychophysiological effects of total/ partial sleep deprivation or extended wakefulness have been welldocumented ${ }^{18}$. There is a substantial variability of the results in sensitivity to sleep loss across the three cognitive domains. For example, the largest performance decrements are observed for measures of sustained attention and working memory ${ }^{19}$.

We have hypothesized that sleep fragmentation compared to sleep efficiency may have a negative impact on the working memory. Accordingly, we investigated the effects of sleep fragmentation and sleep stage on the three domains of cognitive function, i.e., the working memory, executive function, and sustained attention in healthy adults.

\section{MATERIAL AND METHODS}

\section{Subjects}

Twenty-eight healthy adults (18 males and 10 females; mean age $27.8 \pm 15.5$ years) were enrolled in this study. None of the subjects had any history of neurological disorder, substance abuse, head injury, or major physical illness nor were they prescribed any psychotropic medications at the time of the study. This study (No. 270098) was approved by the ethics committee of Chubu University. Written informed consent was obtained from all participants after the nature of the study and procedures involved were fully explained.

\section{Polysomnography (PSG)}

After one night's acclimation, the subjects underwent the standard PSG with Alice 5 (Philips Respironics, Murrysville, PA) in accordance with the Manual of American Academy of Sleep Medicine (version 2.1) ${ }^{20}$. The PSG was conducted in the sleep laboratory at Chubu University. For each subject, lights out clock time was determined according to their habitual bedtime. Wake up time had to be adjusted to be the subjects' needs until lights on clock time at 7:00 a.m. We obtained recordings of 6-channel electroencephalography $\left(\mathrm{F}_{3}-\mathrm{M}_{2}, \mathrm{~F}_{4}-\mathrm{M}_{1}, \mathrm{C}_{3}-\mathrm{M}_{2}, \mathrm{C}_{4}-\mathrm{M}_{1}\right.$, $\mathrm{O}_{1}-\mathrm{M}_{2}$, and $\mathrm{O}_{2}-\mathrm{M}_{1}$ electrodes), right and left electrooculogram, submental electromyograms, and electrocardiograms. We also used a microphone to record snoring and a sensor to monitor body position. Subsequently, we calculated the total sleep time (TST), sleep efficiency [TST/total recording time $($ TRT) $\times 100$, $\%$ ], \%stage wakefulness (W) [W / sleep period time (SPT) $\times$ 100, \%], \%stage REM, \%stage N1, \%stage N2, and \%stage N3.

The sleep stage in every 30 seconds of 1 epoch was scored according the criteria of the Manual of American Academy of Sleep Medicine (version 2.1) ${ }^{20}$. An epoch of stage N1 was scored if the majority of the epoch met the criteria for stage N1 (electroencephalogram showing low-amplitude, mixed-frequency electroencephalogram activity) in the absence of evidence for another sleep stage. An epoch of stage N2 was scored if the majority of the epoch met the criteria for stage N2 ( $K$ complex, sleep spindle). An epoch of stage N3 was scored when $\geq 20 \%$ of an epoch consists of slow wave activity. An epoch of stage REM was scored if the majority of the epoch met the criteria for stage REM (rapid eye movements, low chin electromyogram tone, sawtooth waves, transient muscle activity $)^{20}$. Moreover, we evaluated the wake after sleep onset (WASO) and arousal index to quantify sleep fragmentation. Sleep onset was defined as the start of the first epoch scored as any stage in 30-second other than stage W. The WASO was defined as the sum of all waking hours during the sleep period and includes all wake activity, including time out of bed. Arousal was scored during stage N1, N2, N3 or REM if there was an abrupt shift of electroencephalogram frequency including alpha, theta and/or frequencies greater than 16 $\mathrm{Hz}$ (but not spindles) that lasted at least 3 seconds, with at least 10 seconds of stable sleep preceding the change. The arousal index was defined as the number of arousals per hour ${ }^{20}$.

\section{Cognitive function tests}

In the morning after undergoing PSG, the subjects performed the N-back task ${ }^{14,15}$, WCST ${ }^{16}$, and CPT-IP ${ }^{17}$ to assess working memory, executive function, and sustained attention, respectively.

\section{N-back task}

The participants underwent the N-back task to assess their working memory. In this task, they were required to update 
the mental set continually while responding to the stimuli (i.e., numbers) which were previously seen ${ }^{14,21,22}$. Each test comprised the 14 trials, each of which had a stimulus duration of $0.4 \mathrm{~s}$, an inter-stimulus interval of $1.4 \mathrm{~s}$, and $0-, 1$-, and 2 -back conditions. The stimuli consisted of numbers $(2,4,6$, or 8$)$ shown in random sequence, which were displayed at the points of a diamondshaped box ${ }^{21}$. Participants were required to respond to the stimuli using the numeric keypad of a computer. Performance was measured as the $\%$ correct (Hits + Correct Rejections/Total Stimuli $\times 100)$ and mean reaction time for correct hits on the keypad. In the N-back tasks for assessing working memory, the study participants were asked to indicate the timing when the presented number was the same as a series of number stimuli which were previously displayed.

\section{WCST}

The WCST (WCST-Keio F-S version, Japanese Stroke Data Bank, Japan) was used for assessing their executive functions, including the abilities of reasoning abstract and shifting cognitive strategies in response to changing environmental contingencies ${ }^{16,23}$. We measured the category achievement of the number of categories among a maximum of eight categories, for which six consecutive correct responses were required ${ }^{24}$.

\section{CPT-IP}

The CPT-IP (Biobehavioral Technologies, Inc., New York, NY, USA) was used for assessing the sustained attention or vigilance, as described previously ${ }^{17}$. A series of four-digit stimuli was presented for a period of $50 \mathrm{~ms}$, with an interstimulus interval of $950 \mathrm{~ms}$. Each session comprised 150 trials, among which 30 target trials were required to respond. Two sessions (first and second test) were conducted, and the results of the second test were adopted. Performances were assessed using the signal detection index $d$-prime, which was a measure of discriminability computed from "hits" and "false alarms".

\section{STATISTICAL ANALYSIS}

All data were expressed as mean \pm standard deviation. We performed the Pearson's correlation analyses followed by multiple regression analysis based on a stepwise forward selection method to determine the independent parameters that correlated with the 0-, 1-, and 2-back tasks, WCST, and CPT-IP in relation to \%stage REM, \%stage N1, \%stage N2, and $\%$ stage N3 or TST, sleep efficiency, WASO, and arousal index. A probability value of 0.05 was considered statistically significant. All statistical analyses were performed using the SPSS version 25.0 (IBM Corporation, Armonk, New York, USA).

\section{RESULTS}

The PSG findings and parameters by the N-back task, WCST, and CPT-IP were summarized in Table 1.

\section{Correlation between the sleep parameters and N-back task}

The percentage of correct answers on the 2-back task was significantly correlated with \%stage REM, \%stage N1,
Table 1. PSG findings and parameters by the N-back task, WCST, and CPT-IP

\begin{tabular}{lc}
\hline Parameters & \\
\hline PSG & $383.2 \pm 64.4$ \\
TST (min) & $89.6 \pm 9.3$ \\
Sleep efficiency (\%TRT) & $6.0 \pm 7.2$ \\
Stage W (\%SPT) & $16.7 \pm 6.6$ \\
Stage REM (\%SPT) & $20.0 \pm 14.2$ \\
Stage N1 (\%SPT) & $51.5 \pm 14.9$ \\
Stage N2 (\%SPT) & $5.7 \pm 5.4$ \\
Stage N3 (\%SPT) & $28.3 \pm 35.8$ \\
WASO (min) & $14.2 \pm 13.5$ \\
Arousal index (/h) & \\
N-back task & \\
0-back task & $99.4 \pm 2.0$ \\
\% corrects & $511.4 \pm 99.5$ \\
Reaction time (ms) & \\
1-back task & $97.0 \pm 4.6$ \\
\% corrects & $426.0 \pm 210.6$ \\
Reaction time (ms) & \\
2-back task & $87.0 \pm 13.0$ \\
\% corrects & $436.9 \pm 224.1$ \\
Reaction time (ms) & \\
WCST & $5.7 \pm 0.8$ \\
Category achievement & \\
CPT-IP & $2.5 \pm 0.9$ \\
Signal detection index $(d$-prime) & \\
D. & \\
\hline
\end{tabular}

Data are expressed as mean \pm standard deviation. PSG $=$ Polysomnography; TST $=$ Total sleep time; $\mathrm{TRT}=$ Total recording time; $\mathrm{SPT}=$ Sleep period time; $\mathrm{WASO}=$ Wake after sleep onset; WCST $=$ Wisconsin card sorting test; CPT-IP = Continuous performance test-identical pairs.

and \%stage N2 (\%stage REM: $r=0.505, p=0.006 ; \%$ stage N1: $r=-0.637, p<0.001 ; \%$ stage N2: $r=0.670, p<0.001)$, and \%stage REM and \%stage N2 were the significant factors (\%stage REM: $\beta=0.559, \quad p=0.025 ; \%$ stage N2: $\beta=1.010, \quad p=0.039)$ (Table 2). Multiple regression analysis including the stepwise forward selection method revealed that \%stage $\mathrm{N} 2$ was the most significant factor (\%stage N2: $\beta=0.670, p<0.001$ ). The percentage of correct answers on the 2-back task was also significantly correlated with TST, sleep efficiency, WASO, and arousal index (TST: $r=0.492, p=0.008$; sleep efficiency: $r=0.622$, $p<0.001$; WASO: $r=-0.721, p<0.001$; arousal index: $r=-0.656$, $p<0.001)$, and WASO was the significant factor $(\beta=-2.086$, $p=0.007$ ) (Table 3) (Figure 1). Multiple regression analysis based on a stepwise forward selection method including \%stage REM, \%stage N1, \%stage N2, TST, sleep efficiency, WASO, and arousal index revealed that WASO was the most significant factor of the percentage of correct answers on the 2-back task $(\beta=-0.721, p<0.001)$.

The reaction time on the 1 -back task was significantly correlated with \%stage REM, \%stage N1, and \%stage N2 (\%stage REM: $r=-0.425, \quad p=0.024 ; \%$ stage N1: $r=0.589$, $p=0.001 ; \%$ stage N2: $r=-0.637, p<0.001)$, and \%stage REM and \%stage $\mathrm{N} 2$ were the significant factors (\%stage REM: $\beta=-0.529, p=0.046 ; \%$ stage N2: $\beta=-1.276, p=0.017)$. The 
Table 2. Relationships among parameters by the N-back task, WCST, and CPT-IP and sleep stages.

\begin{tabular}{|c|c|c|c|c|c|c|c|c|}
\hline & \multicolumn{2}{|c|}{ Simple correlation analysis } & \multicolumn{2}{|c|}{ Multiple regression analysis } & \multicolumn{2}{|c|}{ Simple correlation analysis } & \multicolumn{2}{|c|}{ Multiple regression analysis } \\
\hline & $r$ & $p$ & $\beta$ & $p$ & $r$ & $p$ & $\beta$ & $p$ \\
\hline 0 -back task & $\%$ corrects & & & & Reaction time & & & \\
\hline Stage REM & 0.282 & 0.145 & 0.148 & 0.645 & -0.246 & 0.206 & -0.394 & 0.196 \\
\hline Stage N2 & -0.231 & 0.237 & -0.780 & 0.224 & -0.516 & 0.005 & -1.103 & 0.072 \\
\hline Stage N3 & 0.252 & 0.195 & -0.033 & 0.922 & -0.035 & 0.862 & -0.148 & 0.638 \\
\hline Stage N1 & -0.288 & 0.137 & -0.155 & 0.866 & 0.589 & 0.001 & -1.003 & 0.142 \\
\hline Stage N2 & 0.244 & 0.212 & 0.090 & 0.896 & -0.637 & $<0.001$ & -1.276 & 0.017 \\
\hline Stage N3 & 0.166 & 0.397 & 0.039 & 0.915 & -0.214 & 0.273 & -0.313 & 0.247 \\
\hline 2-back task & $\%$ corrects & & & & Reaction time & & & \\
\hline Stage REM & 0.505 & 0.006 & 0.559 & 0.025 & -0.507 & 0.006 & -0.690 & 0.015 \\
\hline Stage N1 & -0.637 & $<0.001$ & 0.616 & 0.327 & 0.511 & 0.005 & -0.815 & 0.253 \\
\hline Stage REM & 0.186 & 0.343 & -0.294 & 0.359 & & & & \\
\hline Stage N1 & -0.290 & 0.134 & -1.808 & 0.039 & & & & \\
\hline Stage N2 & 0.220 & 0.260 & -1.109 & 0.086 & & & & \\
\hline Stage N3 & -0.020 & 0.920 & -0.706 & 0.043 & & & & \\
\hline CPT-IP & $d$-prime & & & & & & & \\
\hline Stage REM & -0.163 & 0.408 & -0.021 & 0.951 & & & & \\
\hline Stage N1 & 0.206 & 0.292 & 0.100 & 0.914 & & & & \\
\hline Stage N2 & -0.126 & 0.524 & -0.011 & 0.988 & & & & \\
\hline Stage N3 & -0.218 & 0.266 & -0.150 & 0.687 & & & & \\
\hline
\end{tabular}

WCST $=$ Wisconsin card sorting test CPT-IP $=$ Continuous performance test-identical pairs.

Table 3. Relationships among parameters by the N-back, WCST, and CPT-IP and TST, sleep efficiency, WASO, and arousal index.

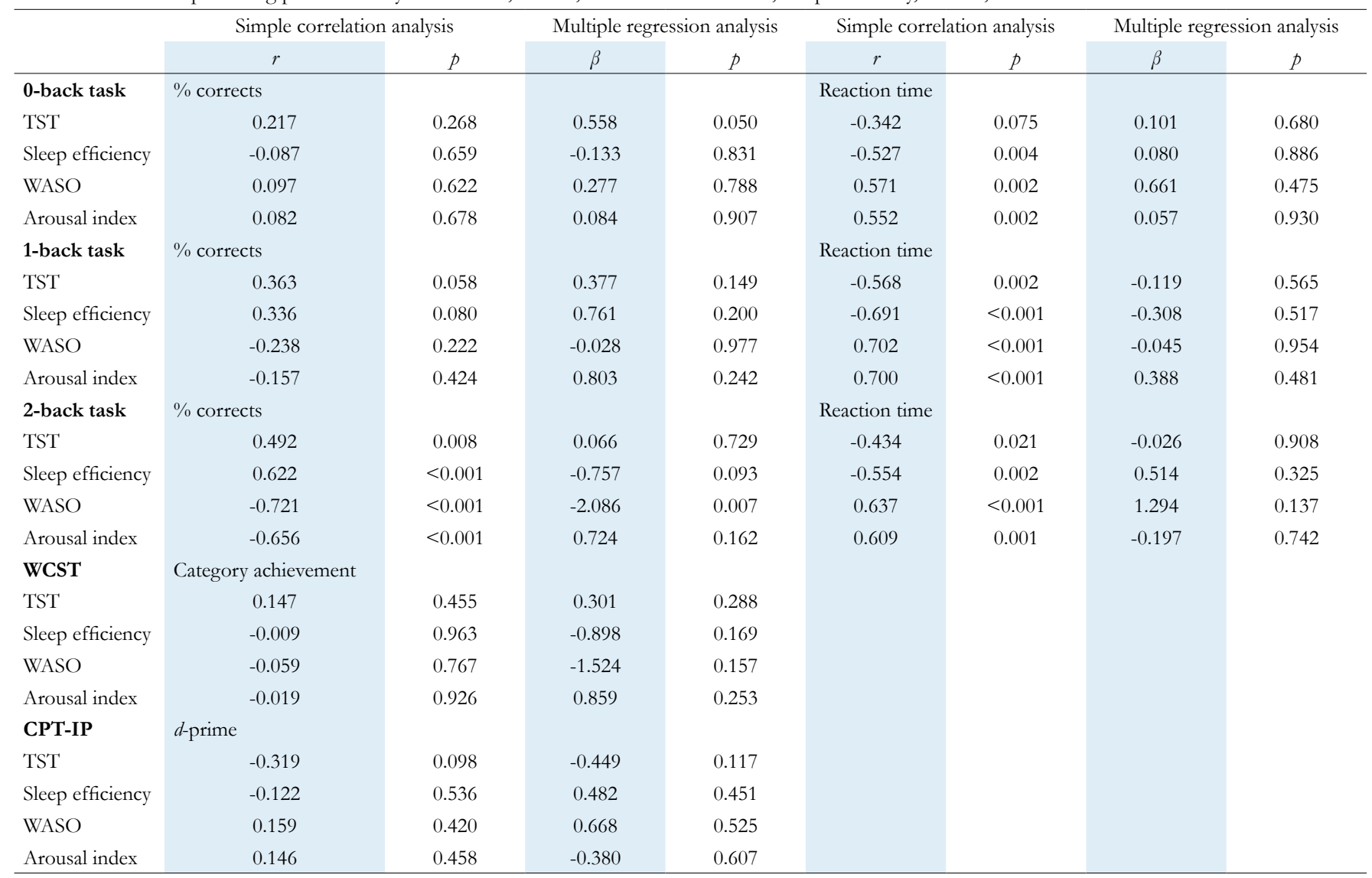

TST $=$ Total sleep time; WASO $=$ Wake after sleep onset; WCST $=$ Wisconsin card sorting test; CPT-IP $=$ Continuous performance test-identical pairs. 


\section{Case 1}

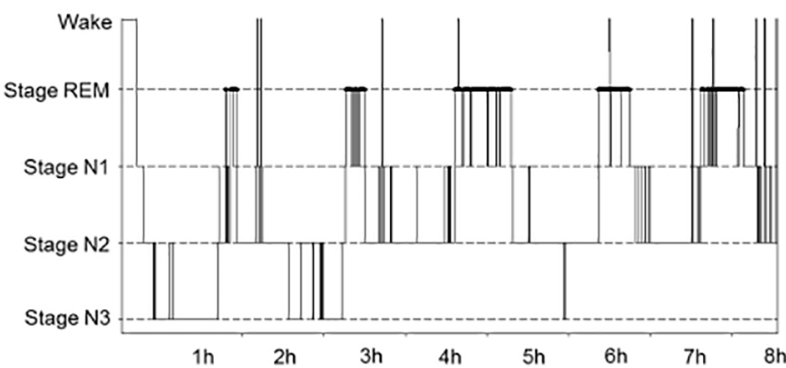

Case 2

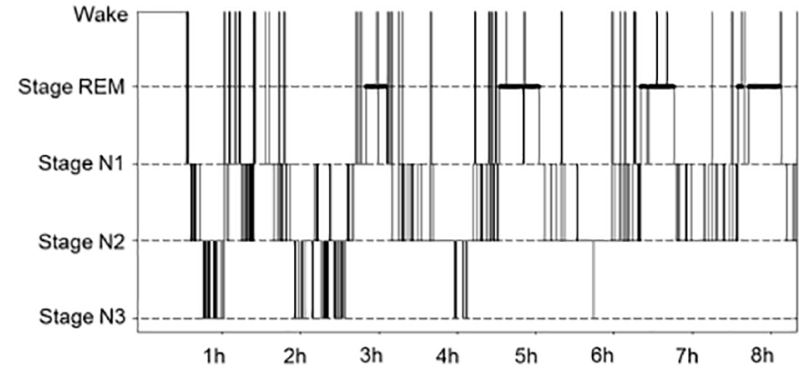

\begin{tabular}{lcc}
\hline & Case 1 & Case 2 \\
\hline TST (min) & 465.5 & 435.0 \\
$\begin{array}{l}\text { Sleep efficiency } \\
\text { (\%TRT) }\end{array}$ & 96.6 & 86.1 \\
Stage W (\%SPT) & 1.0 & 7.0 \\
Stage REM (\%SPT) & 21.2 & 19.9 \\
Stage N1 (\%SPT) & 10.2 & 23.9 \\
Stage N2 (\%SPT) & 53.0 & 44.1 \\
Stage N3 (\%SPT) & 14.7 & 5.2 \\
WASO (min) & 5.5 & 32.5 \\
Arousal index (/h) & 6.2 & 18.6 \\
\% corrects on the & 100.0 & 85.7 \\
2-back task & & \\
\hline
\end{tabular}

Figure 1. PSG hypnograms in representative case. WASO and arousal index were higher and the percentage of correct answers on the 2-back task was lower in case 2 compared to case 1.

TST $=$ Total sleep time; $\mathrm{TRT}=$ Total recording time; $\mathrm{SPT}=$ Sleep period time; WASO $=$ Wake after sleep onset.

reaction time on the 2-back task was significantly correlated with \%stage REM, \%stage N1, and \%stage N2 (\%stage REM: $r=-0.507, p=0.006 ; \%$ stage N1: $r=0.511, p=0.005 ; \%$ stage N2: $r=-0.523, p=0.004)$, and \%stage REM was the significant factor $(\beta=-0.690, p=0.015)$ (Table 2).

\section{Correlation between the sleep parameters and WCST or CPT-IP}

The WCST category achievement and CPT-IP $d$-prime score were not correlated with \%stage REM, \%stage N1, $\%$ stage N2, \%stage N3, TST, sleep efficiency, WASO, and arousal index.

\section{DISCUSSION}

We found that increased WASO and a decrease in \%stage $\mathrm{N} 2$ were associated with the lower percentages of correct answers on the 2-back task, and that WASO was the most significant factor influencing the percentages of correct answers on the 2-back task among three domains (working memory, executive function, and sustained attention) of cognitive function. Our findings suggest that increased WASO and a decrease in \%stage $\mathrm{N} 2$ have a negative effect on the working memory.

Sleep fragmentation plays a vital role in the progression of cognitive decline by promoting endothelial dysfunction ${ }^{25}$ and inflammation ${ }^{26}$. Chronic sleep fragmentation model confirmed that preserved sleep quality in addition to sleep duration is essential for cognitive performance ${ }^{27-29}$. There are substantial changes with aging in the overall sleep quality, slow wave sleep, spindle density, and sleep fragmentation ${ }^{30-32}$. Sleep disturbance is a common symptom among young adults and is associated with such factors as decreased quality of life, chronic somatic diseases, and mental illnesses ${ }^{33}$. A previous study using actigraphy among older community-dwelling people showed that a longer TST and greater WASO were associated with lower cognitive performance ${ }^{34}$. Moreover, sleep fragmentation concomitant with surges in blood pressure and heart rate has been shown to result in daytime sleepiness that impairs psychomotor function even in healthy individuals ${ }^{35}$. Thus, increased WASO and frequent arousals lead to sleep fragmentation and poor sleep quality, which are likely to precipitate the cognitive decline in the long run.

In the present study, the percentage of correct answers on the 2-back task was significantly correlated with \%stage N2. The reaction times on 0 - and 1-back tasks were closely correlated with the \%stage $\mathrm{N} 2$, which reflects visual-speed processing in synchrony with commands of motor output from the premotor area ${ }^{36}$. Stage N2 spindle density was reported to be a marker of neural connectivity of the brain, which was essential for maintaining the general cognitive function ${ }^{37}$. Schabus et al. $(2006)^{38}$ divided sleep spindles into two categories: fast and slow. Slow spindles $(<13 \mathrm{~Hz})$ predominate over the frontal cortical areas, whereas fast spindles $(>13 \mathrm{~Hz})$ prevail over the parietal and central areas $^{39}$. The spindles are associated with activity in the hippocampus ${ }^{38}$. They are temporally coupled with oscillations in the sharp wave ripple of the hippocampus during sleep ${ }^{40}$. Recently, an animal study demonstrated that working memory was associated with hippocampal neuronal activity ${ }^{4}$. Therefore, we believe that stage N2 may provide us with critical information for working memory. Future studies should address potential mechanisms underlying the relationship between \%stage N2 and working memory.

We showed that the percentage of correct answers on the 2-back task significantly correlated with \%stage REM. A recent PSG study in 58 healthy middle-aged and older adults demonstrated that REM sleep duration was associated with better learning potential of verbal memory, which suggest that longer REM sleep duration may be a marker of acetylcholine system integrity ${ }^{42}$. Moreover, the ability to maintain working 
memory was associated with REM duration and TST in the daytime nap in 80 healthy college students ${ }^{43}$. The effect of sleep loss on the working memory was found to relate to the degraded neural activation of the prefrontal cortex (PFC) and functional connectivity with other brain regions ${ }^{44}$. Although the mechanisms of the dynamic changes of the PFC activities from NREM to REM have remained unclear, REM sleep appears to play an important role in the selective activation and deactivation of PFC during sleep. Hence, sufficient REM sleep could preserve the enhanced working memory.

In this study, the percentage of correct answers on the 2-back task was not significantly correlated with \%stage N3. Working memory provides a temporary storage space and the information necessary for processing tasks and acts as the bridge between the instantaneous and long-term memory systems in the human brain ${ }^{45}$. Using electroencephalography, a recent study in healthy male adults demonstrated that higher slow wave activity in the frontoparietal regions predicted a better working memory ${ }^{46}$. A PSG study in healthy middle-aged and older participants reported that slow wave density and slow wave slope were correlated with verbal fluency performance, but no significant correlations were found between 2-back performance and any sleep variables ${ }^{42}$. An intervention study on three weeks of working memory training in male children and adolescents showed that the increase in slow wave activity was correlated with cognitive training-induced plasticity in a region known to be involved in working memory performance ${ }^{47}$. The methodology of evaluation and ages of the subjects may influence the different effects of stage N3 on working memory.

The category achievement by the WCST was not correlated with \%stage REM, \%stage N2, and WASO. The category achievement was 6 in $57.1 \%$ of all participants, which may have created a ceiling effect due to the ease of completing the WCST in healthy adults. Regarding the difference between the WCST and N-back task of the frontal tasks, the N-back task is a test for working memory processes ${ }^{14,15,48}$, and the WCST is a test for executive function ${ }^{16}$. Hence, the differential role of the N-back task and WCST could explain the differences in our results for the two tasks. Moreover, the CPT-IP $d$-prime score, which reflects sustained attention, was not correlated with \%stage REM, \%stage N2, and WASO in this study. A previous PSG study showed that there were no significant correlations between the parameter by CPT-IP and sleep variables in healthy middle-aged and older participants ${ }^{42}$, which seems consistent with our findings.

Our study had a few limitations. First, our study population was relatively small. Second, we could not investigate the gender differences in the effect of sleep fragmentation on cognitive function owing to our small sample size. The sexes did not differ significantly on the N-back task in 93 healthy young subjects aged 17 to 32 years $^{49}$. However, insufficient sleep may enhance anxiety in women. Women are more susceptible to the emotional consequences of sleep deprivation and sleep disruption than men ${ }^{50,51}$. Third, the current study is a cross- sectional study and it is not possible to determine whether WASO is the cause of cognitive decline. To assess the effects of sleep fragmentation on working memory, we could not evaluate the effects of arousals on working memory when subjects were randomly divided into two groups, with one group using, for example, auditory stimulation to induce sleep fragmentation and the other group allowing subjects to sleep uninterruptedly. Moreover, a clearer index of fragmentation is the number of arousals, even if TST is not changed. Another one could be the probability of transition from NREM to stage $\mathrm{W}^{52}$. Intervention trials with larger sample sizes may be required to elucidate the effect of sleep stage and sleep fragmentation on the working memory, executive function, and sustained attention in healthy adults of both genders.

\section{CONCLUSION}

Among the three domains of cognitive function, increased sleep fragmentation and a decrease in \%stage N2 were associated with worse working memory. Evaluation of the major domains of cognitive function along with PSG analysis could yield practical and theoretical implication for cognitive impairment.

\section{ACKNOWLEDGMENTS}

The study was supported by the Japan Society for the Promotion of Science (KAKENHI Grant No. 25282210) and Chubu University research grant (No. 19M27A1).

\section{REFERENCES}

1. Diekelmann S, Born J. The memory function of sleep. Nat Rev Neurosci. 2010 Feb;11(2):114-26.

2. Banks S, Dinges DF. Behavioral and physiological consequences of sleep restriction. J Clin Sleep Med. 2007 Aug;3(5):519-28.

3. Kronholm E, Sallinen M, Suutama T, Sulkava R, Era P, Partonen T Self-reported sleep duration and cognitive functioning in the general population. J Sleep Res. 2009 Dec;18(4):436-46.

4. Fernandez-Mendoza J, He F, Calhoun SL, Vgontzas AN, Liao D, Bixler EO. Objective short sleep duration increases the risk of all-cause mortality associated with possible vascular cognitive impairment. Sleep Health. 2020 Feb;6(1):71-8.

5. Zohar D, Tzischinsky O, Epstein R, Lavie P. The effects of sleep loss on medical residents' emotional reactions to work events: a cognitiveenergy model. Sleep. 2005 Jan;28(1):47-54.

6. Rauchs G, Desgranges B, Foret J, Eustache F. The relationships between memory systems and sleep stages. J Sleep Res. 2005 Jun;14(2):123-40.

7. Goerke M, Müller NG, Cohrs S. Sleep-dependent memory consolidation and its implications for psychiatry. J Neural Transm (Vienna). 2017 Feb;124(Suppl 1):163-78.

8. Stickgold R. Sleep-dependent memory consolidation. Nature. 2005 Oct;437(7063):1272-8.

9. Stickgold R, Whidbee D, Schirmer B, Patel V, Hobson JA. Visual discrimination task improvement: a multi-step process occurring during sleep. J Cogn Neurosci. 2000 Mar;12(2):246-54.

10. Walker MP, Brakefield T, Morgan A, Hobson JA, Stickgold R. Practice with sleep makes perfect: sleep dependent motor skill learning. Neuron. 2002 Jul;35(1):205-11.

11. Kato K, Iwamoto K, Kawano N, Noda Y, Ozaki N, Noda A. Differential effects of physical activity and sleep duration on cognitive function in young adults. J Sport Health Sci. 2018 Apr;7(2):227-36.

12. Miyata S, Noda A, Ozaki N, Hara Y, Minoshima M, Iwamoto K, et al. Insufficient sleep impairs driving performance and cognitive function. Neurosci Lett. 2010 Jan;469(2):229-33.

13. Miyata S, Noda A, Iwamoto K, Kawano N, Banno M, Tsuruta Y, et al Impaired cortical oxygenation is related to mood disturbance resulting from three nights of sleep restriction. Sleep Biol Rhythms. 2015 Oct;13(4):387-94. 
14. Callicott JH, Bertolino A, Mattay VS, Langheim FJ, Duyn J, Coppola R, et al. Physiological dysfunction of the dorsolateral prefrontal cortex in schizophrenia revisited. Cereb Cortex. 2000 Nov;10(11):1078-92.

15. Owen AM, McMillan KM, Laird AR, Bullmore E. N-back working memory paradigm: a meta-analysis of normative functional neuroimaging studies. Hum Brain Mapp. 2005 May;25(1):46-59.

16. Alvarez JA, Emory E. Executive function and the frontal lobes: a metaanalytic review. Neuropsychol Rev. 2006 Mar;16(1):17-42.

17. Cornblatt BA, Risch NJ, Faris G, Friedman D, Erlenmeyer-Kimling L. The continuous performance test, identical pairs version (CPT-IP): I. new findings about sustained attention in normal families. Psychiatry Res. 1988 Nov;26(2):223-38.

18. Krause AJ, Simon EB, Mander BA, Greer SM, Saletin JM, GoldsteinPiekarski AN, et al. The sleep-deprived human brain. Nat Rev Neurosci. 2017 Jul;18(7):404-18.

19. Lim J, Dinges DF. A meta-analysis of the impact of short-term sleep deprivation on cognitive variables. Psychol Bull. 2010 May;136(3):375-89.

20. Berry RB, Brooks R, Gamaldo CE, Harding SM, Lloyd RM, Marcus CL, et al. The AASM manual for the scoring of sleep and associated events: rules, terminology and technical specifications, version 2.1 . Darien: American Academy of Sleep Medicine (AASM); 2014.

21. Callicott JH, Mattay VS, Bertolino A, Finn K, Coppola R, Frank JA, et al. Physiological characteristics of capacity constraints in working memory as revealed by functional MRI. Cereb Cortex. $1999 \mathrm{Jan} / \mathrm{Feb}$;9(1):20-6.

22. Jacola LM, Willard VW, Ashford JM, Ogg RJ, Scoggins MA, Jones MM, et al. Clinical utility of the N-back task in functional neuroimaging studies of working memory. J Clin Exp Neuropsychol. 2014 Oct;36(8):875-86.

23. Tomida K, Takahashi N, Saito S, Maeno N, Iwamoto K, Yoshida K, et al. Relationship of psychopathological symptoms and cognitive function to subjective quality of life in patients with chronic schizophrenia. Psychiatry Clin Neurosci. 2010 Jan;64(1):62-9.

24. Banno M, Koide T, Aleksic B, Yamada K, Kikuchi T, Kohmura K, et al. A case control association study and cognitive function analysis of neuropilin and tolloid-like 1 gene and schizophrenia in the Japanese population. PLoS One. 2011;6(12):e28929.

25. Sauvet F, Florence G, Van Beers P, Drogou C, Lagrume C, Chaumes C, et al. Total sleep deprivation alters endothelial function in rats: a nonsympathetic mechanism. Sleep. 2014 Mar;37(3):465-73.

26. Irwin MR, Wang M, Campomayor CO, Collado-Hidalgo A, Cole S. Sleep deprivation and activation of morning levels of cellular and genomic markers of inflammation. Arch Intern Med. 2006 Sep;166(16):1756-62.

27. Ramesh V, Nair D, Zhang SX, Hakim F, Kaushal N, Kayali F, et al. Disrupted sleep without sleep curtailment induces sleepiness and cognitive dysfunction via the tumor necrosis factor- $\alpha$ pathway. J Neuroinflammation. 2012;9:91.

28. Baud MO, Magistretti PJ, Petit JM. Sustained sleep fragmentation affects brain temperature, food intake and glucose tolerance in mice. J Sleep Res. 2013 Feb;22(1):3-12.

29. Petit JM, Gyger J, Burlet-Godinot S, Fiumelli H, Martin JL, Magistretti PJ. Genes involved in the astrocyte-neuron lactate shuttle (ANLS) are specifically regulated in cortical astrocytes following sleep deprivation in mice. Sleep. 2013 Oct;36(10):1445-58.

30. Bishop NA, Lu T, Yankner BA. Neural mechanisms of ageing and cognitive decline. Nature. 2010 Mar;464(7288):529-35.

31. Bliwise DL, Ansari FP, Straight LB, Parker KP. Age changes in timing and 24-hour distribution of self-reported sleep. Am J Geriatr Psychiatry. 2005 Dec;13(12):1077-82.
32. Mander BA, Winer JR, Walker MP. Sleep and human aging. Neuron. 2017 Apr;94(1):19-36.

33. Chen X, Gelaye B, Williams MA. Sleep characteristics and health-related quality of life among a national sample of American young adults: assessment of possible health disparities. Qual Life Res. 2014 Mar;23(2):613-25.

34. Blackwell T, Yaffe K, Ancoli-Israel S, Redline S, Ensrud KE, Stefanick ML, et al. Association of sleep characteristics and cognition in older communitydwelling men: the MrOS sleep study. Sleep. 2011 Oct;34(10):1347-56.

35. Martin SE, Wraith PK, Deary IJ, Douglas NJ. The effect of nonvisible sleep fragmentation on daytime function. Am J Respir Crit Care Med. 1997 May;155(5):1596-601.

36. Kuriyama K, Mishima K, Suzuki H, Aritake S, Uchivama M. Sleep accelerates the improvement in working memory performance. J Neurosci. 2008 Oct;28(40):10145-50.

37. Fogel SM, Smith CT. The function of the sleep spindle: a physiological index of intelligence and a mechanism for sleep-dependent memory consolidation. Neurosci Biobehav Rev. 2011 Apr;35(5):1154-65.

38. Schabus M, Hödlmoser K, Gruber G, Sauter C, Anderer P, Klösch G, et al Sleep spindle-related activity in the human EEG and its relation to general cognitive and learning abilities. Eur J Neurosci. 2006 Apr;23(7):1738-46.

39. Schabus M, Dang-Vu TT, Albouy G, Balteau E, Boly M, Carrier J, et al Hemodynamic cerebral correlates of sleep spindles during human non-rapid eye movement sleep. Proc Natl Acad Sci U S A. 2007 Aug;104(32):13164-9.

40. Clemens Z, Mölle M, Eross L, Jakus R, Rásonyi G, Halász P, et al. Finetuned coupling between human parahippocampal ripples and sleep spindles. Eur J Neurosci. 2011 Feb;33(3):511-20.

41. Sasaki T, Piatti VC, Hwaun E, Ahmadi S, Lisman JE, Leutgeb S, et al. Dentate network activity is necessary for spatial working memory by supporting CA3 sharp-wave ripple generation and prospective firing of CA3 neurons. Nat Neurosci. 2018 Feb;21(2):258-69.

42. Lafortune M, Gagnon JF, Martin N, Latreille V, Dubé J, Bouchard M, et al. Sleep spindles and rapid eye movement sleep as predictors of next morning cognitive performance in healthy middle-aged and older participants. J Sleep Res. 2014 Apr;23(2):159-67.

43. Lau EYY, Wong ML, Lau KN, Hui FWY, Tseng CH. Rapid-eyemovement-sleep (REM) associated enhancement of working memory performance after a daytime nap. PLoS One. 2015;10(5):e0125752.

44. Nofzinger EA. Functional neuroimaging of sleep. Semin Neurol. 2005 Mar;25(1):9-18.

45. Baddeley A. The episodic buffer: a new component of working memory?. Trends Cogn Sci. 2000 Nov;4(11):417-23.

46. Ferrarelli F, Kaskie R, Laxminarayan S, Ramakrishnan S, Reifman J, Germain A. An increase in sleep slow waves predicts better working memory performance in healthy individuals. Neuroimage. 2019 May;191:1-9.

47. Pugin F, Metz AJ, Wolf M, Achermann P, Jenni OG, Huber R. Local increase of sleep slow wave activity after three weeks of working memory training in children and adolescents. Sleep. 2015 Apr;38(4):607-14.

48. Baddeley A. Working memory. Science. 1992 Jan;255(5044):556-9.

49. Evans KL, Hampson E. Sex differences on prefrontally-dependent cognitive tasks. Brain Cogn. 2015;93:42-53.

50. Birchler-Pedross A, Schröder CM, Münch M, Knoblauch V, Blatter K, Schnitzler-Sack C, et al. Subjective well-being is modulated by circadian phase, sleep pressure, age, and gender. J Biol Rhythms. 2009 Jun;24(3):232-42.

51. Van Der Helm E, Gujar N, Walker MP. Sleep deprivation impairs the accurate recognition of human emotions. Sleep. 2010 Mar;33(3):335-42.

52. Ferri R, Drago V, Aricò D, Bruni O, Remington RW, Stamatakis K, et al. The effects of experimental sleep fragmentation on cognitive processing. Sleep Med. 2010 Apr;11(4):378-85. 